\title{
Multichannel auditory brainstem implantation: the Australian experience
}

\author{
R.J.S. Briggs* ${ }^{\dagger}$, P. Fagan ${ }^{\ddagger}$, M. Atlas ${ }^{\ddagger}$, A. H. Kaye ${ }^{\dagger}$, J. Sheehy ${ }^{\ddagger}$, R. Hollow*, S. ShaW**, \\ G. M. Clark*
}

\begin{abstract}
The multichannel auditory brainstem implant (ABI) provides the potential for hearing restoration in patients with neurofibromatosis type 2 (NF2). Programmes for auditory brainstem implantation have been established in two Australian centres. Eight patients have been implanted under the protocol of an international multi-centre clinical trial. Three patients had ABI insertion at the time of first side tumour removal, four at second side tumour removal and one after previous bilateral surgery where there was some residual tumour. The translabyrinthine approach was used in all cases. Successful positioning of the electrode array was achieved in seven of eight patients, all of whom achieved auditory perception with electrical stimulation. Intra-operative electrically evoked auditory brainstem response testing was successful in four patients and was useful in confirming correct electrode position. In six cases postoperative psychophysical and auditory perception testing demonstrated that useful auditory sensations were achieved. Five of these patients regularly used the implant. In one patient electrode placement was unsuccessful and only non-auditory sensations occurred on stimulation. In the remaining patients nonauditory sensations were minimal and avoidable by selective electrode programming. Auditory brainstem implantation should be considered in patients with NF2. The greatest benefit is seen in patients without debilitating disease who have non-aidable hearing in the contralateral ear.
\end{abstract}

Key words: Brain Stem; Prosthesis Implantation; Neurofibromatosis 2; Australia

\section{Introduction}

Patients with neurofibromatosis type 2 (NF2) who have bilateral acoustic neuromas may suffer total hearing impairment due to the growth or surgical removal of their tumours. Limited hearing restoration is now a realistic goal in these patients using direct electrical stimulation of the cochlear nucleus with the auditory brainstem implant (ABI). The initial studies of cochlear nucleus implantation were performed by William House and William Hitselberger $^{1}$ using a variety of prototype electrode arrays and a percutaneous transmission system based on the 3M-Cochlear Implant. Stimulation of the electrodes produced auditory sensation in most patients with results similar to a single channel cochlear implant. Following collaboration between Cochlear Limited and the House Ear Institute, a fully implantable, multi-electrode prosthesis was developed based on the Nucleus 22 channel cochlear implant. The electrode array is placed over the surface of the cochlear nucleus within the lateral recess of the fourth ventricle at the time of translabyrinthine acoustic neuroma removal. Stimulation is via a transcutaneous coil system with a variety of speech processing strategies available, depending on the results of electrode mapping. In most cases multiple channels are available for auditory stimulation without non-auditory sideeffects and the SPEAK speech processing strategy is used. 2,3

The electrode array used consists of eight platinum disc electrodes mounted on a silastic carrier. The improved auditory perception results in patients using this fully implantable multichannel ABI has led to the establishment of a multicentre international trial controlled by the US Food and Drug Administration. Programmes for auditory brainstem implantation have been established in two Australian centres, St. Vincent's Hospital in Sydney and the Royal Melbourne Hospital in co-operation with the University of Melbourne and the Royal Victorian Eye and Ear Hospital in Melbourne. The Nucleus eight electrode ABI has been used and this paper reports the results of eight patients implanted since 1995.

\section{Materials and methods}

Patients with NF2 and bilateral acoustic neuromas in whom surgical tumour removal was planned, were 
considered for auditory brainstem implantation. Institutional and ethics committee consent for the trial was obtained. Suitable patients were Englishspeaking, aged 15 years or older, assessed to be psychologically suitable, to be compliant with the research protocol and to have realistic expectations. The translabyrinthine approach was used for tumour removal in all cases. The only modification was to create a larger than usual anteriorly based scalp flap so that the incision was positioned behind the proposed site of the receiver stimulator and antenna. After acoustic neuroma removal the foramen of Luschka and lateral recess of the fourth ventricle was identified by retraction of the cerebellar flocculus and identifying where the stump of the VIIIth nerve entered the brainstem superior to the glossopharyngeal nerve. Positive identification of the lateral recess was confirmed by the presence of choroid plexus and egress of cerebrospinal fluid. The electrode array with its Dacron mesh backing was then gently inserted into the lateral recess with the electrodes facing superiorly.

After the electrode array was positioned and the receiver stimulator package secured, intra-operative electrically evoked auditory brainstem response (EABR) testing was performed. A sterile transmitting coil was placed over the antenna of the receiver stimulator and the ABI was stimulated using the transduction coil. The stimulus amplitude was gradually increased and the resulting stimulus artefact monitored. Subsequently the first part of the sweep was electronically blanked out to eliminate the stimulus artefact and allow recognition of the EABR wave form. When a potential EABR wave form was identified, the polarity of the electrical stimulus was reversed, in which case if the wave form was a neural response, it remained unchanged. Continuous electromyographic monitoring of cranial nerves VII, IX and $\mathrm{X}$ was also performed. If an EABR wave form was not identified or a myogenic response or cranial nerve stimulation occurred, then the electrode array was repositioned until an EABR wave form was identified, or until it was felt that optimal anatomical electrode placement had been achieved.

Six weeks after surgery initial stimulation of the implant was performed. Psychophysical testing was used to assess the stimulation threshold and comfort levels on each electrode. The presence of any auditory or non-auditory sensation was noted in both bipolar and monopolar stimulation modes. Where possible a pitch ranking of electrodes with auditory sensations was performed.

Patients were then fitted with a speech processor using the 'SPEAK' speech processing strategy. A MAP was created using those electrodes with only auditory percept or very minimal non-auditory sensation. A bipolar or monopolar stimulation mode was used depending on the auditory response and presence of non-auditory sensations.

After initial programming, MAPping and experience with the ABI, comprehensive auditory perception testing was performed with tests of consonants, vowels, sentences, monosyllabic words and environmental sound recognition. Assessment was performed at three monthly intervals for the first year and then annually thereafter. The sound effects recognition test (SERT) was used for environmental sound testing. NU-CHIPS is the North Western University Childrens Perception of Speech test and the Monosyllable, Spondee, Trochee, Polysyllable (MSTP) test is a closed set test of word and stressed syllable recognition. For sentences, the City University of New York (CUNY) and the Central Institute for the Deaf (CID) sentence tests were used. Testing was performed for vision alone, sound alone and for sound plus vision.

\section{Results}

Since September 1995 eight patients have undergone auditory brainstem implantation, three in Melbourne and five in Sydney. There were five females and three males with ages ranging from 17 to 45 years. Three patients underwent auditory brainstem implantation at the time of first side acoustic neuroma removal. One of these patients had a profound hearing loss in the contralateral ear whilst two had useful hearing in the contralateral side, one with near normal thresholds and one with a severe loss requiring a hearing aid. Four patients underwent implantation at the time of the second side tumour removal and one patient had had previous bilateral tumour removals via retrosigmoid approaches. He had a small amount of residual tumour in the internal auditory canal and at the brainstem on the left side. Tumour sizes ranged from $1 \mathrm{~cm}$ to over 4 $\mathrm{cm}$. In each case electrode placement was performed after tumour removal. Intra-operative EABR monitoring was assessed to be successful in four cases. In these cases repeatable responses were obtained and the wave form was noted to be stable when the polarity of the electric stimulus was reversed. In two of these four cases the intra-operative evoked ABR influenced final electrode placement as the electrode array was repositioned (usually with progressive insertion into the lateral recess) until the optimal wave form was obtained without activation of the cranial nerve monitors. In one case intra-operative EABR monitoring was considered inconclusive and in three cases no repeatable response was obtained.

Post-operative recovery in all patients was as expected following the acoustic neuroma surgery. Complications occurred such as temporary or permanent facial paralysis and, in one case, deep venous thrombosis requiring anticoagulation. None of these problems was thought to be contributed to by the auditory brainstem implant.

Successful electrode array placement and subsequent auditory perception was achieved with stimulation in all except one patient. This patient was a 31-year-old male in whom the ABI was inserted at the time of second side tumour removal. $\mathrm{He}$ had a very large tumour with brainstem compression and presumably the electrode array was not adequately retained within the lateral recess. At the time of post-operative initial stimulation only 
non-auditory sensations were obtained. Subsequent surgical re-exploration was attempted in the hope of repositioning the electrode array. This was unsuccessful, however, due to the presence of dense fibrous tissue reaction involving the electrode array and brainstem. Another patient who underwent a more recent implantation has not been MAPped and does not use the device. This patient, a 45 year-old male has multiple intracranial and spinal tumours and underwent auditory brainstem implantation at the time of first side acoustic neuroma removal. Although sound perception is achieved with stimulation with no non-auditory side-effects, the patient has a thick skin flap overlying the receiver stimulator antenna and when initially fitted with a speech processor the MAP was not heard. It is hoped that the use of a high powered Spectra 22 speech processor will overcome this problem. This patient has good contralateral hearing and he has currently deferred further attempts at programming whilst he undergoes treatment for his spinal tumours.

The remaining six patients have been successfully fitted with speech processors using the SPEAK strategy and all have completed comprehensive auditory perception testing. In three patients the monopolar stimulation mode is used and in three the bipolar mode is used. All patients except one, have some non-auditory side-effects such as facial tingling, facial nerve stimulation, vertigo or somatic trunk or leg sensation. In each case the non-auditory percepts are avoided by programming only those electrodes with auditory sensation alone. The number of electrodes used in the MAP ranges from three to eight with the average being five. Some electrodes are used in a variety of combinations thereby increasing the number of channels available for the MAP. Five patients wear the auditory brainstem implant regularly, four on a full time basis and one part time. The sixth patient, who also has aidable contralateral hearing does not wear the implant for daily use. The five patients who do use their implant all have no remaining hearing. They achieve benefit from recognition of environmental sound and as an aid to lip-reading with implant use in daily communication. No patient has achieved any open set speech understanding.
The results of auditory perception testing are summarized in Table I. For closed set testing of word and stressed syllable recognition, all patients scored well above chance level. For audition alone with CUNY sentence testing, no patient had any significant score. When comparing vision alone to sound and vision however, most patients demonstrated a significant improvement in scores demonstrating the aid to speech understanding that is achieved when using the ABI. The speech perception scores shown in Table I represent the results of the most recent testing. The duration since time of implantation varies from one to three years. Some patients have shown progressive improvement in their speech perception scores with increased duration of implant use whilst others appear to have remained relatively stable compared with the initial three month post-operative results.

\section{Discussion}

The Australian experience with auditory brainstem implantation has demonstrated that significant benefit in terms of restoration of useful auditory perception can be achieved for NF2 patients with bilateral profound or total hearing loss. Useful perception of environmental sound and an aid to lip-reading is obtained that is helpful in the patient's communication and daily activities. In this series no patient has achieved any open set speech understanding.

Although this series is small, it is clear from the results that the patients who will benefit the most and who will use their ABI regularly are those who have no useful residual hearing and are otherwise active and in generally good health. The patient in this series who uses the ABI only part-time is becoming progressively debilitated by her disease. Initially after the $A B I$ surgery she returned to work and found the ABI beneficial. Unfortunately she is becoming increasingly weak, has now stopped work and with the decreased activity and interaction uses her implant less and less. Presumably this is because of the relatively limited benefit in terms of improved speech perception and lack of open set speech understanding. Patients who are active and indepen-

TABLE I

AUDITORY BRAINSTEM IMPLANTATION: SPEECH PERCEPTION RESULTS

\begin{tabular}{lccl}
\hline Patient & NU-CHIPS* (chance 25\%) & MSTP* (chance 8\% word 33\% stress) & CUNY sentences* \\
\hline VM & $50 \%$ & $50 \%$ Word & $19 \%$ Vision alone \\
& & $71 \%$ Stress & $56 \%$ Sound and vision \\
AM & $70 \%$ & $83 \%$ Word & $35 \%$ Vision alone \\
& & $96 \%$ Stress & $67 \%$ Sound and vision \\
JC & $100 \%$ & $58 \%$ Word & $13 \%$ Vision alone \\
& & $75 \%$ Stress & $65 \%$ Sound and vision \\
EN & $98 \%$ & $33 \%$ Word & $80 \%$ Vision alone \\
& & $83 \%$ Stress & $85 \%$ Sound and vision \\
NL & $96 \%$ & $25 \%$ Word & $68 \%$ Vision alone \\
KM & $50 \%$ & $63 \%$ Stress & $72 \%$ Sound and vision \\
& & $46 \%$ Word & $69 \%$ Vision alone \\
MB & ABI & $67 \%$ Stress & $82 \%$ Sound and vision \\
JD & ABI & Not mapped &
\end{tabular}

*See text for details 
dent find that the perception of environmental sound and the aid to lip reading that is achieved with the use of the implant can be very valuable. Patients who are not independent are perhaps less likely to value the relatively limited degree of sound perception obtained. We believe that this should be taken into account when considering recommending auditory brainstem implantation for patients with the more aggressive form of NF2.

The presence of other intracranial or spinal tumours is not in itself a contra-indication to auditory brainstem implantation. Our first patient implanted has subsequently undergone removal of the contralateral acoustic neuroma with no associated surgical problem and no change in the function of her implant. Other patients have had cervical and spinal tumours removed with no problems associated with surgery or subsequent implant function.

The other groups of patients who also do not utilize the potential benefit of their implant are those who have useful hearing in the contralateral ear. Two patients in this series do not use the implant for this reason. Performing auditory brainstem implantation at the time of first side tumour removal where there is useful contralateral hearing is reasonable if there is the likelihood of imminent loss of that hearing. For example, where there is a large contralateral tumour or progressive hearing impairment already. For patients where there is a small tumour and good hearing in the contralateral ear the arguments against, probably outweigh those arguments for, implantation at the time of first side tumour removal. The patient is not likely to use or achieve any benefit from a first side ABI in the foreseeable future. Any experience with programming and using the ABI is unlikely to alter the longterm benefit achieved if, and when, the contralateral hearing is lost. There is a small but real possibility of device failure. There is likely to be, and already have been, advances in design that can provide improved performance and benefit over a current device. There is potential difficulty with safe removal of the electrode array from the lateral recess should revision be required. Finally, our experience has confirmed that successful auditory brainstem implantation is possible after previous tumour removal surgery. These issues should be understood and decision about first side implantation made on an individual basis considering the potential longer term benefit of successful first side implantation. Also the potential for tumour removal with cochlear nerve preservation must be considered, as in this situation, cochlear implantation is likely to be more beneficial than brainstem implantation if hearing is lost.

Another potential contra-indication to auditory brainstem implantation is where there is a very large tumour and associated brainstem distortion on the side of planned tumour removal and implantation. The one case in this series where electrode array placement was unsuccessful was where there was a very large tumour. The potential for difficulty with electrode array placement and retention should be considered when recommending implantation for patients with large tumours.

In summary, the Australian experience has demonstrated that, for appropriately selected patients, multichannel auditory brainstem implantation can provide limited but useful hearing restoration.

\section{References}

1 Edgerton BJ, House WF, Hitselberger WE. Hearing by cochlear nucleus stimulation in humans. Ann Otol Rhinol Laryngol 1982;91(Suppl 9):117-24

2 Skinner M, Clark G, Whitford L, Seligman PM, Staller SJ, Shipp DB, et al. Evaluation of a new spectral peak coding strategy for the nucleus 22 channel cochlear implant system. Am J Otol 1994;15 (Suppl 2):15-27

3 Otto S, Staller S. Multichannel auditory brainstem implant: Case studies comparing fittings, strategies and results. Ann Otol Rhinol Laryngol 1995;104(Suppl 166):36-9

Address for correspondence:

Robert J. S. Briggs,

Department of Otolaryngology,

Royal Victorian Eye and Ear Hospital,

32 Gisborne Street,

East Melbourne 3002,

Victoria

Australia.

E-mail: rjbriggs@netspace.net.au 


\section{University Library}

\section{- M M N E R VA A gateway to Melbourne's research publications}

Minerva Access is the Institutional Repository of The University of Melbourne

Author/s:

Briggs, R. J. S.;Fagan, P.;Atlas, M.;Kaye, A. H.;Sheehy, J.;Hollow, R.;Shaw. S.;Clark, Graeme M.

Title:

Multichannel auditory brainstem implantation: the Australian experience

Date:

2000

Citation:

Briggs, R. J. S., Fagan, P., Atlas, M., Kaye, A. H., Sheehy, J., Hollow, R., et al. (2000). Multichannel auditory brainstem implantation: the Australian experience. The Journal of Laryngology \& Otology, December, 114(Suppl. 27), 46-49.

Persistent Link:

http://hdl.handle.net/11343/28752 